\section{Hepatic Failure in a Patient with Metastatic Lung Cancer after Experiencing Partial Response during Chemotherapy and Immunotherapy}

\section{Abstract}

We present the case of a 62-year-old patient who developed dyspnoea, chest and dorsal pain. The history was taken, finds patients was smoker and has high blood pressure, chronic obstructive pulmonary disease and sleep apnea syndrome. So, the patients followed by Complementary tests (tomography, bronchoscopy and endoscopic ultrasound. he was diagnosed with lung adenocarcinoma stage IV (T4N3M1b bone and pulmonary lesions, no liver masses) without target driver. He was stared chemotherapy and immunotherapy in clinical trial with tumor response but liver failure. We performed a differential diagnosis to discern the etiology of the fulminant hepatitis.

Keywords: Lung adenocarcinoma; Chemotherapy; High blood pressure; Immunotherapy; Hepatic failure; Disease progression; Hepatitis; Lung cancer; Autoimmunity

Received: November 16, 2017; Accepted: December 01, 2017; Published: December 06, 2017

\section{Introduction}

Lung cancer is the most frequently diagnosed cancer with high mortality, being the leading cause of death from cancer in men [1]. A survival of $15 \%$ at 5 years is since more than $70 \%$ of patients are diagnosed in advanced stages [2,3]. Metastatic dissemination occurs by hematogenous route and by lymphatic route [4]. Nowadays there are many treatments used for those tumors and have changed the paradigm of the disease (with benefit in progression free survival and overall survival terms) [5]. Some of those drugs are novel so the interpretation and the correct management of the side effects is for the moment a challenge raising the doubt in some occasions between toxicity or disease progression [6]. It is important to focus on awareness of Lung Cancer physicians to improve early detection rates.

\section{Case Presentation}

A 62-year-old patient was admitted to the hospital because dyspnoea, chest and dorsal pain. The patient was smoker and had history of high blood pressure, chronic obstructive pulmonary disease and sleep apnea syndrome. Complementary test was done (tomography, bronchoscopy and endoscopic ultrasound) disease [9].
Maria Galan Gramaje ${ }^{1}$, Carme Garcia Lorenzo ${ }^{1}$, Ana Isabel De Hita Santabaya ${ }^{2}$ and Juan Coves Sarto ${ }^{1}$

1 Department of Medical Oncology, Hospital Son Llatzer, Spain

2 Department of Pathological Anatomy, Hospital Son Llatzer, Spain

\section{Corresponding author: Maria Galan}

”galan38@hotmail.com

Department of Medical Oncology, Hospital Son Llatzer, Spain.

Tel: 0034664400622

Citation: Galan M, Lorenzo CG, Santabaya AIDH, Sarto JC (2017) Hepatic Failure in a Patient with Metastatic Lung Cancer after Experiencing Partial Response during Chemotherapy and Immunotherapy. Arch Cancer Res. Vol.5 No.4:163

$[7,8]$ and he was diagnosed with lung adenocarcinoma stage IV (T4N3M1b bone and pulmonary lesions) without target driver (wild type EGFR, no rearrangement ALK, no mutation ROS1, PDL1 positive 50\%). With (good performance status, he began treatment with chemotherapy (carboplatin-nabpaclitaxel) and immunotherapy atezolizumab) in a clinical trial. After 3 cycles of treatment, abdominal and chest tomography were practiced with tumor response. One cycle after, he experienced progressive increase of transaminases, so we stopped the treatment.

At first, we reviewed the diagnostic tests as well as those required for the clinical trial. At the diagnosis, an abdominal ultrasound was performed without alterations as well as an anodyne computerized axial tomography; in addition, a reassessment tomography had recently been performed without liver findings except hepatomegaly that could translate into chronic liver

Likewise, prior to starting the treatment vitric serologies were performed (HIV, HBV, HCV and EBV) all of which were negative except $\mathrm{HBs}$ antibodies positivity, being this result compatible with vaccination against $\mathrm{HBV}$. After the worsening of the liver 
profile, new viral serologies and autoimmunity were requested, those being negative. In addition, the patient had a normal proteinogram.

Because of the anodyne findings, a new tomography was requested which showed maintenance of the tumor response but worsening of the hepatomegaly with periportal edema and ascites (Figure 1).

All the drugs that the patient regularly took were also reviewed, as well as the drugs that he received in the clinical trial. The patient denied alcohol consumption as a possible cause of hepatopathy and thus of cholestasis.

No other analytical determinations were requested given the absence of compatible clinical symptoms and given that finally because of the radiological deterioration of the hepatomegaly a liver biopsy was requested and was diagnostic.

\section{Discussion}

Lung cancer is the most frequently diagnosed cancer and the leading cause of death from cancer in men, although in women it is the leading cause of death from cancer in developed countries and the second one in less developed countries. It presents a high mortality, with a survival of $15 \%$ at 5 years and this is since more than $70 \%$ of patients are diagnosed in advanced stages. Within the lung carcinoma is distinguished the small cell variant (assuming less than 15\% of the cases at present) and the nonsmall cell variant (the most frequent histological subtypes are first adenocarcinoma (45\%-50\%) followed by squamous cell carcinoma). Metastatic dissemination occurs by hematogenous route and also by lymphatic route. The most frequent metastases settle in lung, adrenal, liver, bone and central nervous system. The metastatic liver involvement constitutes $20 \%-35 \%$ of the cases, being able to be symptomatic (abdominal pain, jaundice, fever) or asymptomatic.

The elevation of the liver enzymes can translate acute or chronic hepatitis depending on the severity of the elevation off

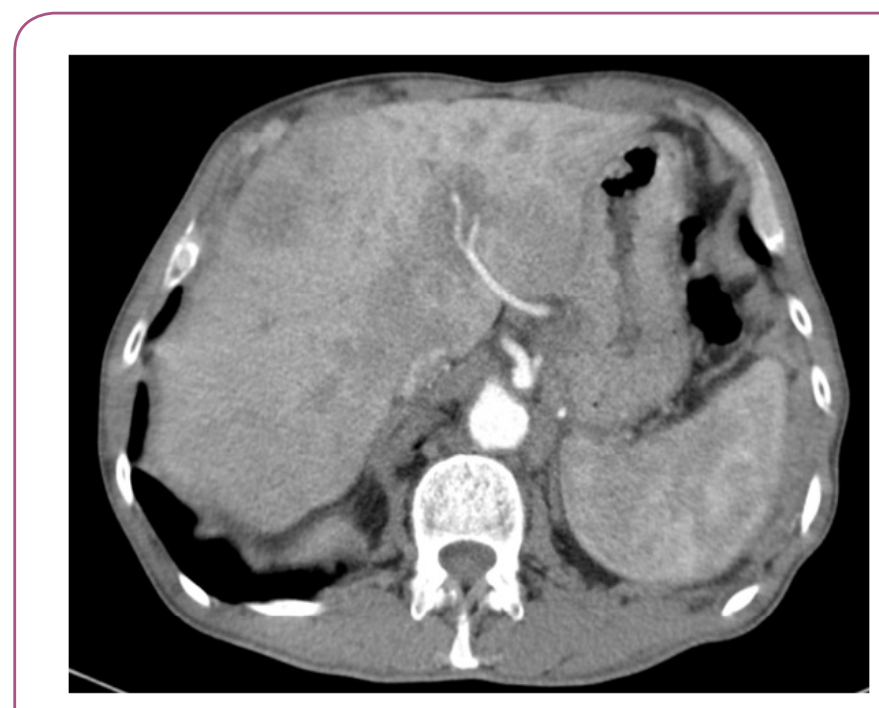

Figure 1 Hepatomegaly with periportal edema.

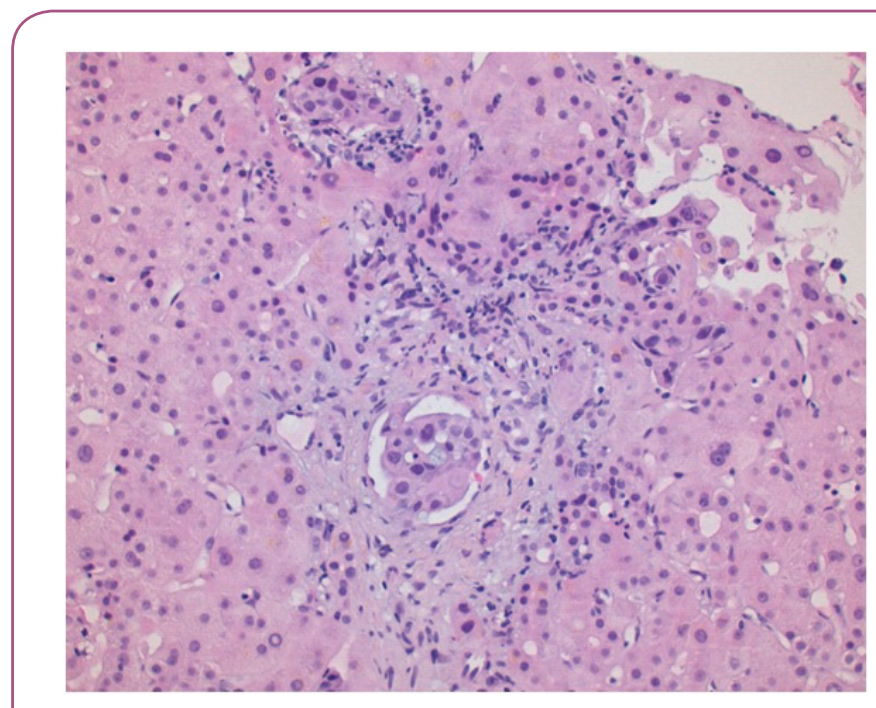

Figure 2 Liver biopsy (Hematoxylin-eosin).

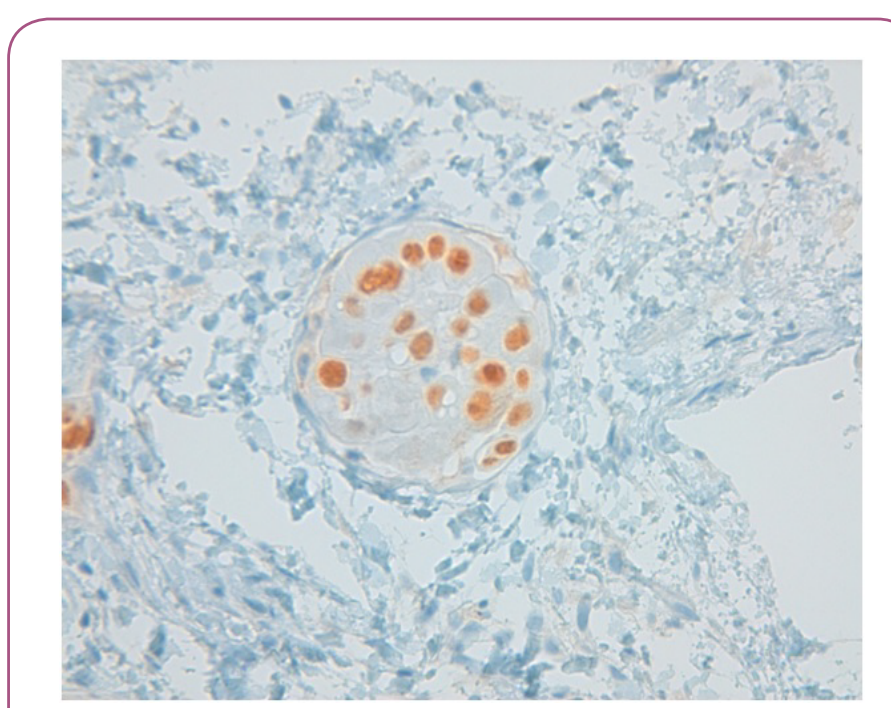

Figure 3 Liver biopsy (Positive TTF1).

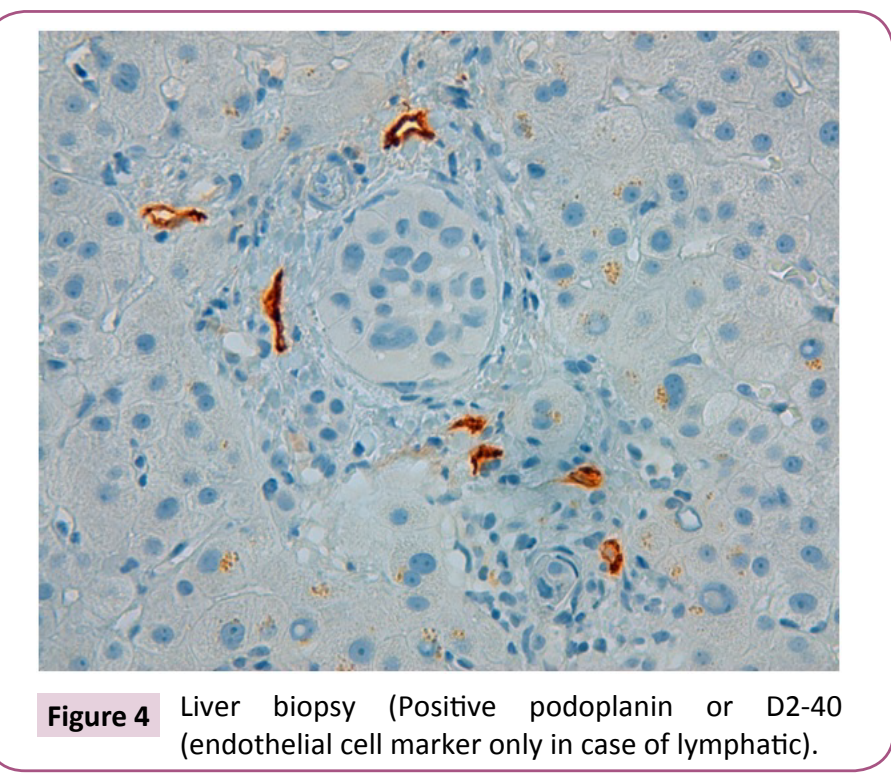


the same as well as time in which said elevation is established. In our case, although the onset of liver enzyme elevation was less than 6 months, it was not a marked elevation compared to normal values. At first, dissociated cholestasis predominated in front of cytolysis. For this reason, new viral serologies were requested, which turned out to be negative, ruling out this cause as probable. Other causes that were ruled out were alcoholism (no history of alcohol consumption), some systemic diseases as well as autoimmune liver diseases (serology for autoimmune hepatitis also was determined and negative). A tomography was also requested to rule out metastatic focal affectation without findings.

As for the drugs that could produce hepatotoxicity, the patient had only taken sporadically some ibuprofen and although there are cases of idiosyncratic hepatitis described by this drug, it was not the initial suspicion given the time it had taken the same and the patient's context.

Regarding chemotherapy, carboplatin is a drug that can cause mild or moderate impairment of liver function tests, especially affecting alkaline phosphatase, but which usually subsides during treatment. Nabpaclitaxel, if administered together with carboplatin, can produce hyperbilirubinemia, although our patient did not present this alteration. Atezolizumab is a humanized monoclonal IgG1 antibody against the programmed cell death protein-ligand 1 (PD-L1) that prevents interaction with PD1 or B7 in activated T lymphocytes and other immune cells, preventing a negative regulation of immunity. The existence

\section{References}

1 Yasufuku K, Chiyo M, Koh E, Moriya Y, lyoda A, et al. (2005) Endobronchial ultrasound guided transbronchial needle aspiration for staging of lung cancer. Lung Cancer 50: 347-354.

2 Jemal A, Siegel R, Ward E, Murray T, Xu J, et al. (2006) Cancer statistics, 2006. CA: CA Cancer J Clin 56: 106-130.

3 Paez JG, Janne PA, Lee JC, Tracy S, Greulich H, et al. (2004) EGFR mutations in lung cancer: Correlation with clinical response to gefitinib therapy. Science 304: 1497-1500.

4 Chambers AF, Groom AC, MacDonald IC (2002) Metastasis: Dissemination and growth of cancer cells in metastatic sites. Nat Rev Cancer 2: 563-572.

5 Molina JR, Yang P, Cassivi SD, Schild SE, Adjei AA (2008) Non-small cell of immuno-related hepatitis has been described in clinical trials, although in percentages below $1 \%$ and although it is not associated with other drugs (even in clinical trials). Therefore, it could not be ruled out that it was toxicity due to immunotherapy.

Given the evolution of the case and without being able to make an accurate clinical diagnosis, diagnostic liver biopsy was requested.

\section{Conclusion}

After liver biopsy the final diagnosis was: Liver parenchyma with lymphovascular invasion due to carcinoma of pulmonary origin (Figures 2 - 4). For this reason, the patient left the study due to progression of the disease with poor outcome and worsening of liver function tests (including coagulation) with subsequent liver failure being the cause of the patient's death.

The diffuse metastatic involvement of the hepatic parenchyma that correlates with hepatic failure is an unusual and extremely rare form of presentation of liver metastases. However, series of cases with rapid progression due to acute hepatic failure have been described after being diagnosed post mortem. Therefore, despite not being the most frequent cause of presentation, we must think that it is a diagnostic option considering even if necessary liver biopsy.

\section{Conflict of Interest}

The authors declare that they have no conflict of interest that competes with any of the contents of the manuscript.

lung cancer: Epidemiology, risk factors, treatment, and survivorship. Mayo Clin Proc 83: 584-594.

6 Suzuki Y, Suda T, Furuhashi K, Suzuki M, Fujie M, et al. (2010) Increased serum kynurenine/tryptophan ratio correlates with disease progression in lung cancer. Lung Cancer 67: 361-365.

7 Coleman FP (1947) Primary carcinoma of the lung, with invasion of the ribs: Pneumonectomy and simultaneous block resection of the chest wall. Ann Surg 126: 156.

8 Muhm JR, Miller WE, Fontana RS, Sanderson DR, Uhlenhopp MA (1983) Lung cancer detected during a screening program using fourmonth chest radiographs. Radiol 148: 609-615.

9 Fraquelli M, Rigamonti C, Casazza G, Conte D, Donato MF (2007) Reproducibility of transient elastography in the evaluation of liver fibrosis in patients with chronic liver disease. Gut 56: 968-973. 